\title{
Cariprazine in Three Special Different Areas: A Real-World Experience
}

\author{
Camilla Gesi ${ }^{1}$ \\ Silvia Paletta ${ }^{2}$ \\ Maria Carlotta Palazzo' \\ Bernardo Dell'Osso',3 \\ Claudio Mencacci' \\ Giancarlo Cerveri ${ }^{2}$ \\ 'Department of Mental Health and \\ Addiction, ASST FBF Sacco, Milan, Italy; \\ ${ }^{2}$ Department of Mental Health and \\ Addiction, ASST Lodi, Lodi, Italy; ${ }^{3}$ School \\ of Medicine University of Milan, Milan, \\ Italy
}

\begin{abstract}
Cariprazine is an antipsychotic medication which received approval from the US Food and Drug Administration for the treatment of schizophrenia in September 2015. Cariprazine is a dopamine $D_{3}$ and $D_{2}$ receptor partial agonist, with a preference for the $D_{3}$ receptor. Furthermore, although to a more limited extent, cariprazine also exhibits partial agonism at the level of 5- $\mathrm{HT}_{1 \mathrm{~A}}$ receptors, thus exerting an antidepressant effect in addition to the antipsychotic effect. The most commonly encountered adverse events are extrapyramidal symptoms and akathisia. Short-term weight gain appears infrequently. Cariprazine is not associated with any clinically meaningful alterations in metabolic variables, prolactin, or ECG QT interval. Cariprazine is also approved for the acute treatment of manic or mixed episodes associated with bipolar I disorder. Clinical trials of cariprazine are ongoing in patients with acute bipolar I depression and as adjunctive treatment to antidepressant therapy in patients with major depressive disorder. In this article, we present some significant clinical cases regarding the use of cariprazine, with the hope that our experience can provide insight or suggestions to be used in clinical practice.
\end{abstract}

Keywords: cariprazine, schizophrenia, bipolar disorder, effectiveness, tolerability

\section{Introduction}

Cariprazine is a novel Second-Generation Antipsychotic (SGA) with antagonistpartial agonist properties at $D_{2}$ and $D_{3}$ receptors, with preferential binding to the $D_{3}$ receptors. ${ }^{1}$ It has been approved by the US Food and Drug Administration (FDA) ${ }^{2}$ and European Medicines Agency (EMA) ${ }^{3}$ for the treatment of adult patients with schizophrenia and for acute treatment of manic/and mixed episodes of Bipolar Disorder (BD) (only FDA). ${ }^{2}$

Currently, SGAs are considered the mainstay of treatment for both acute episodes' management and relapse prevention of schizophrenia. ${ }^{4}$ Moreover, they are increasingly used in different phases of $\mathrm{BD} .^{5}$ Clinical evidence has shown that cariprazine could be especially favorable for patients with predominant negative symptoms with an excellent tolerability profile. In this regard, a recent clinical review has highlighted that, across all studies, the most common side effects of cariprazine across different patient groups were represented by akathisia, extrapyramidal symptoms, insomnia, headache, dizziness, tremor, and gastrointestinal disturbances. $^{6}$

Clinical trials are mandatory to provide solid data about the efficacy and tolerability of pharmacological treatments. However, they may not be representative of the entire patient population, which comprises patients often excluded from 
clinical trials, showing complex clinical presentation and poor response to usual treatments. Thus, real-world clinical cases could help to improve the knowledge of actual effectiveness and tolerability of cariprazine in more difficult clinical scenarios.

The present report includes three clinical cases.

The first one focused on the efficacy of cariprazine on negative symptoms and highlighted the importance of adequate observation time before a beneficial effect could be detected, especially in negative symptomatology and overall functioning.

The second clinical case illustrated cariprazine add-on treatment in a patient with poor response to clozapine. Adding to previous similar reports, we highlighted the potential usefulness and safety of cariprazine in improving antipsychotic response and metabolic tolerability of clozapine.

In the third case we described the use of cariprazine in a subject with Rapid Cycling Bipolar Disorder (RCBD), a condition characterized by a pattern of four or more episodes of mania or depression in one year and associated with worse disease outcome and more severe disability. ${ }^{7,8}$ Given the efficacy shown by cariprazine in $\mathrm{BD}$ and the scarcity of controlled trials in RCBD patient group, the latter case may provide clinicians with some useful hints for dealing with this complex diagnostic area.

\section{Case I}

\section{Efficacy on Positive and Negative Symptoms in Schizophrenia}

Case 1 was a 50-year-old, caucasian man. Since losing his job as a customer service operator, he had been unemployed for many years and currently lives with his parents. He was treated for more than 20 years by a private psychiatrist who diagnosed him with schizoaffective disorder. Over the whole period he received several pharmacological treatments, though struggling to achieve sufficient functioning and subjective wellbeing. Among others, he reported poor positive symptoms control during previous treatment with aripiprazole, ziprasidone and lurasidone, and massive weight gain and hyperglycemia with olanzapine. Cognitive impairment and sedation were also constant with previous antipsychotic trials, and the main referred reason for social and work impairment. About two years ago Case 1 was hospitalized for two weeks upon a rapid worsening of auditory hallucinations, paranoid thoughts, and suicidal ideation. During inpatient treatment, haloperidol and benzodiazepines were added to the former therapy, combining lithium $(900 \mathrm{mg} /$ day) and risperidone ( $6 \mathrm{mg} /$ daily). Upon discharge, Case 1 was referred to public out-patient community service for further monitoring and follow-up. On the first evaluation he was obviously sedated. His behavior was hostile and he still reported residual auditory hallucinations (mentioning voices mainly arising in the evening), which appeared as an echo of thoughts. He no longer presented with frank delusion, but mentioned a sense of inability, low selfesteem and a strong discouragement due to a complex pattern of side effects, including asthenia, daytime sedation, slowness of movement, which he thought were more troublesome for social functioning than hallucinations. Case 1 insisted that his functioning decreased as he was started on lithium, several years before, following a sixmonth trial with an antidepressant that did not ameliorate his severe apathy and abulia. He had preserved basic skills, such as personal hygiene routine, driving the car and using public transport, but could not work or maintain social relationships. Relatives described him as inactive and increasingly isolated from family and friends (he stopped seeing a small group of very supportive friends who usually involved him in weekend trips and he spent most of his time in his bedroom). Upon accurate review of psychopathological history, lithium was stopped and benzodiazepines progressively tapered until discontinuation, with a first improvement in terms of side effects. Subsequently, risperidone and haloperidol were gradually cross-titrated with cariprazine (from 1.5 up to $6 \mathrm{mg} /$ day) over about 6 weeks. After 3 months, cariprazine was reduced to $4.5 \mathrm{mg} /$ day because of mild subjective activation and restlessness. In parallel, the patient's functioning started to gradually improve. A few months later, for the first time in a while, Case 1 joined his friends on a photo trip outside the city and, more importantly, he entered a job placement program which he had refused several times in the past. Since joining the community outpatient service two years ago, symptoms have been stable and Case 1 obtained two job grants and was eventually hired for a part-time job. From time to time he still experiences hallucinations, however, the trustful relationship with the service helps him to sort them out. Case 1 currently shows a more active interest in working and meeting his friends and eventually, his negative symptomatology has remarkably improved. 


\section{Discussion}

We presented the case of a patient with schizoaffective disorder showing significant residual symptoms beyond treatment with lithium and several second generation antipsychotics, who was effectively treated with cariprazine monotherapy. The improvement was especially sound in the negative component, although good response was shown for positive symptoms as well.

This is in line with data from the literature suggesting that second-generation antipsychotics, especially cariprazine and amisulpride, should be preferred over first-generation antipsychotics for the treatment of psychotic conditions with pronounced negative symptoms, as they are associated with better functional outcomes and lower cognitive impairment. ${ }^{9}$ Besides exemplifying the effectiveness of cariprazine already shown in controlled trials and arguably involved in the improvement not only in secondary but also in primary negative symptoms,${ }^{10}$ our case highlights how the decrease in negative symptoms may later be reflected in the improvement in work and social performance. Importantly, a subsequent analysis of the same data demonstrated that cariprazine provides additional quality-adjusted life-years over 54 weeks compared with risperidone. ${ }^{11}$ The persistence of positive symptoms, however, never completely treated with previous therapies, did not prevent a better overall functioning of the patient due to a decrease of the negative symptomatology. We found it especially important to include a detailed timing of improvement milestones achieved by the patients in order to provide rough guidance about the time lapse before a beneficial effect on negative symptoms and overall functioning eventually arose. In agreement with previous real-world recommendation, ${ }^{12}$ our case may encourage clinicians to extend the observation period to at least six months after starting cariprazine and before further evaluation on efficacy is done.

\section{Case 2}

\section{Add-On Cariprazine in}

\section{Treatment-Resistant Schizophrenia Partial Respondent to Clozapine}

Case 2 was a 25 -year-old, Caucasian man, living with his parents and an older brother. The family history of mental disorders is positive (one grandmother suffered from a mood disorder and attempted suicide; an aunt died by suicide). He had no history of any major medical illnesses or treatment. He was born full-term after eutocic delivery and had unremarkable developmental history. At the age of 17 he started to show concentration difficulties and behavioral abnormalities, causing significant impairment in both social and school functioning. He was then prescribed risperidone $2 \mathrm{mg} /$ day, rapidly suspended due to extra pyramidal side effects. At the age of 18 , following the exacerbation of behavioral abnormalities, he was admitted to the inpatient psychiatric clinic and BD was diagnosed. Upon treatment with carbamazepine $400 \mathrm{mg} /$ day, and lithium up to $900 \mathrm{mg} /$ day, he managed to obtain a high school diploma. However, after a few months behavioral abnormalities reappeared, followed by psychomotor agitation, dysphoria, delusional ideation with mystical and grandiose contents and auditory hallucination and interpretativeness. Despite augmentation with several antipsychotics (haloperidol, olanzapine, risperidone, chlorpromazine, aripiprazole) and 6 sessions of electroconvulsive therapy (ECT), positive symptoms did not remit over several months while progressive deterioration of functioning, negative symptoms and social withdrawal also became prominent. Upon the absence of primary mood symptoms, a diagnosis of Schizophrenia was made. After more than a year of treatment, clozapine was introduced and gradually titrated up to $700 \mathrm{mg} /$ day. In the next months, further worsening of positive and negative symptoms was observed, with mystical delusion (he reported seeing demons and being in hell), persecutory behavior, auditory hallucinations, anxiety, and severe withdrawal. Illness insight was absent. The patient was therefore admitted to the psychiatric in-patient unit for clinical and pharmacological monitoring. On entering the psychiatric ward, speech had to be stimulated. Thought was characterized by persecutory delusion and interpretativeness. Fluctuating hallucinatory symptoms created significant anguish and discomfort to the patient. Moreover, a relevant negative component was present, with apathy, lack of psychomotor initiative, anhedonia, social withdrawal. Ongoing treatment included clozapine $600 \mathrm{mg} /$ day, lorazepam $7.5 \mathrm{mg}$ /day, promazine $20 \mathrm{mg}$ /day, atenolol $100 \mathrm{mg} /$ day. As brain MRI showed no abnormalities and a clozapine plasma level of $225 \mathrm{ng} / \mathrm{mL}$ was found (it was not possible to increase the clozapine dose because at doses above $600 \mathrm{mg} /$ day the patient began to experience tachycardia and changes in electrocardiogram), cariprazine was started and gradually titrated from $1.5 \mathrm{mg}$ /day up to $6 \mathrm{mg} /$ day. Case 2 showed a significant improvement in terms of both positive and negative symptoms (Figure 1A and B), with an improvement percentage of $47.8 \%$ and $43.6 \%$ respectively. Delusion and distress decreased and 


\section{A}

140

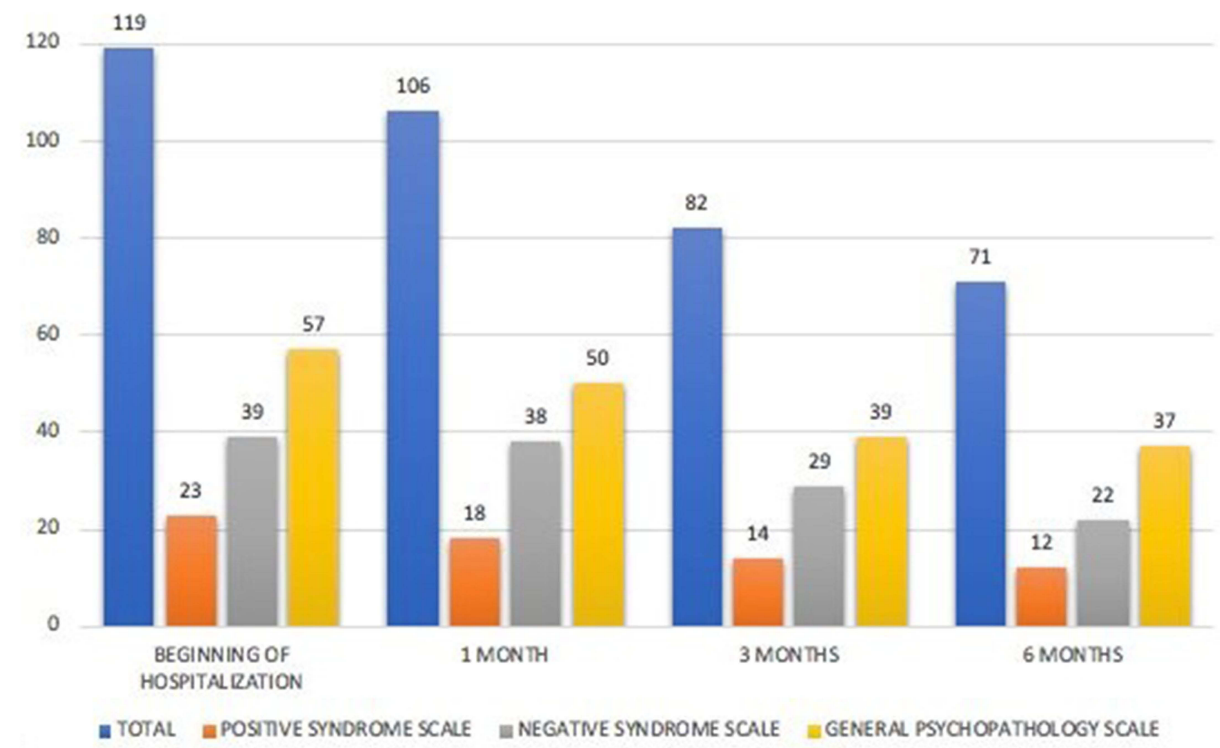

B

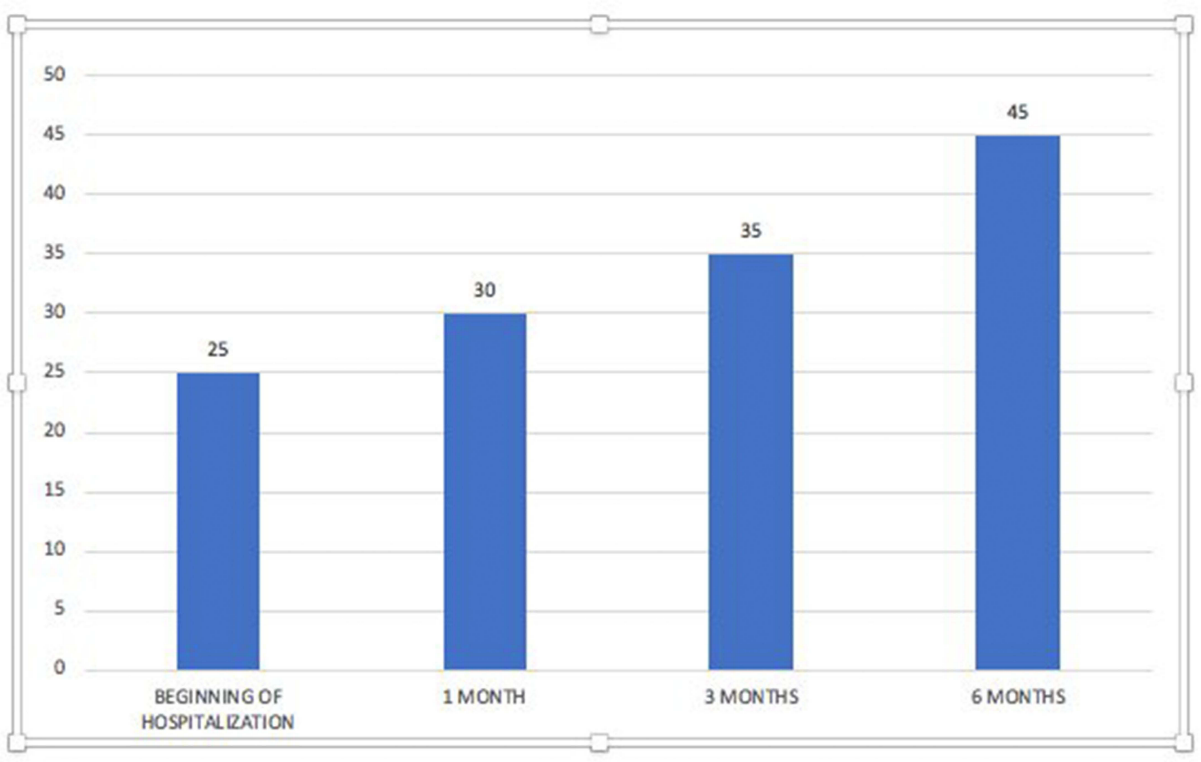

Figure I (A) Clinical course from the beginning of hospitalization up to 6 months of treatment. (B) Clinical course from the beginning of hospitalization up to 6 months of treatment.

Abbreviations: PANSS, Positive and Negative Syndrome Scale; GAF, Global Assessment of Functioning.

auditory hallucinations disappeared. Upon discharge, Case 2 began to attend a community-based day center service where, albeit with significant difficulties, he was involved in a cognitive remediation program and took part in some group activities. Across the following 6 months Case 2 underwent regular clinical monitoring and showed no side effects or metabolic impairment. Instead, BMI showed a slight reduction (from $29 \mathrm{~kg} / \mathrm{m}^{2}$ to $27 \mathrm{~kg} / \mathrm{m}^{2}$ ) and clozapine plasma level never exceeded $250 \mathrm{ng} / \mathrm{mL}$.
Moreover, Case 2 showed increasing willingness to verbalize his emotions and to share his needs with the psychiatric team.

\section{Discussion}

We reported a case of refractory schizophrenia, previously treated with clozapine monotherapy and ECT, that showed a good response to a combination of clozapine and cariprazine. Despite the fact that caution should be used in 
antipsychotic polypharmacy due to the risk of drug interaction and enhanced toxicity, several clozapine augmentation strategies have been reserved for patients with more refractory schizophrenia. ${ }^{13}$ Our case showed a significant improvement in both positive and negative symptoms upon cariprazine augmentation, allowing long-term community-based clinical monitoring and remediation intervention to follow after repeated in-patient treatments. The reduction in both symptom dimensions is consistent with cariprazine binding profile, mainly involving $\mathrm{D}_{2}$ and $\mathrm{D}_{3}$ receptors. $\mathrm{D}_{3}$ mechanism of action of cariprazine is thought to be responsible for negative symptoms' reduction but also for a pro-cognitive effect, resulting in the improvement of executive deficits, and cognitive impairment. Furthermore, although to a more limited extent, cariprazine also exhibits partial agonism at the level of $5 \mathrm{HT}_{1 \mathrm{~A}}$ receptors, thus exerting an antidepressant effect in addition to the antipsychotic effect. These mechanisms may explain the positive impact of the observed cariprazine-clozapine combination. Importantly, regular monitoring of clozapine plasma level, never exceeding $250 \mathrm{ng} / \mathrm{mL}$ over the entire follow-up period, allowed us to rule out that improvement was due to the enhancement of clozapine concentration based on a pharmacokinetic mechanism, as hypothesized in a previous series of two cases focusing on the beneficial use of a cariprazine-clozapine combined treatment. ${ }^{14}$ Noteworthy, the patient did not show any extrapyramidal, cardiovascular or metabolic side effects, showing instead a slight reduction in BMI. Our case thus confirms cariprazine's effectiveness and tolerability and suggests that it might be considered in addition to clozapine in patients with refractory schizophrenia.

\section{Case 3}

\section{A Case of Rapid-Cycling Bipolar Disorder Treated with Cariprazine}

Case 3 was a 58-year-old, Caucasian, married women, with a part-time office job, living with her husband and a 26-year-old daughter. She had a personal medical history of hypothyroidism and no family history of mental disorders. She had a major depression episode at age 29 years. At 32, following her first manic episode in the postpartum period, she was diagnosed with BD type I and started lithium treatment at $900 \mathrm{mg} /$ day. Thereafter, she suffered from recurrent depressive and manic/hypomanic episodes, with no regular pattern of relapses. As add-on treatments, several mood stabilizers were alternatively added to the lithium regimen across the whole period (aripiprazole $10 \mathrm{mg}$ /day, quetiapine up to $600 \mathrm{mg} /$ day, lamotrigine up to $125 \mathrm{mg}$ /day). At the age of 53, during perimenopause, she was diagnosed with a chronic tubulointerstitial nephropathy and lithium treatment was discontinued. From then on, the disorder featured a rapid-cycling course, with eight to nine mood episodes over the course of a year. Yet, both depressive and expansive episodes showed a reduction in length and symptom severity. During this time Case 3 received stable treatment with $1 \mathrm{mg} /$ day of valproate in combination with second-generation antipsychotics (asenapine $10 \mathrm{mg} /$ day, ziprasidone $120 \mathrm{mg} /$ day, quetiapine up to $900 \mathrm{mg} /$ day), with no significant changes in the clinical pattern. In the last eighteen months a daily mood chart was implemented showing a depression-(hypo)mania-free interval cycle pattern, overall lasting about 40 days (Figure 2). About six months ago, due to lack of efficacy, cognitive impairment and constipation, quetiapine was progressively tapered over a 20-day-period and $1.5 \mathrm{mg} /$ day of cariprazine was offered to the patient, upon giving information about its off-label use. Shortly after quetiapine was discontinued, Case 3 started to show dysphoric mood, motor activation and insomnia which rapidly disappeared after titration of cariprazine up to $3 \mathrm{mg} /$ day. Since then, Case 3 displayed a further ten-day depressive episode and a couple of subclinical mood swings and then fulfilled her first 4-month period of euthymic mood over the last five years (last visit's Young Mania Rating Scale score $=4$, Hamilton Depression Rating Scale score=4). Both she and her husband also reported significant improvement in cognitive functioning. Her combination treatment currently includes valproate ( $1 \mathrm{~g} /$ day), cariprazine $(3 \mathrm{mg} /$ day), lorazepam ( $2 \mathrm{mg} /$ day), and L-thyroxine $(75 \mathrm{mcg} /$ day $)$.

\section{Discussion}

Rapid cycling (RC) affects up to $33 \%$ of bipolar patients ${ }^{15}$ and is associated with worse outcomes of the mood disorder, greater comorbidity and polypharmacy. ${ }^{16}$ Data concerning the pharmacological treatment of RC patients are scant and in several instances conflicting. Lithium, valproate, quetiapine, and olanzapine seem to have comparable but poor - maintenance properties for RC patients while some evidence supports the use of lamotrigine in RC BD type II but not type I. ${ }^{7,17,18}$ Literature also suggests that monotherapy with a single mood stabilizer is often ineffective and patients may require a combination of mood stabilizers to achieve mood stability, despite a combination 


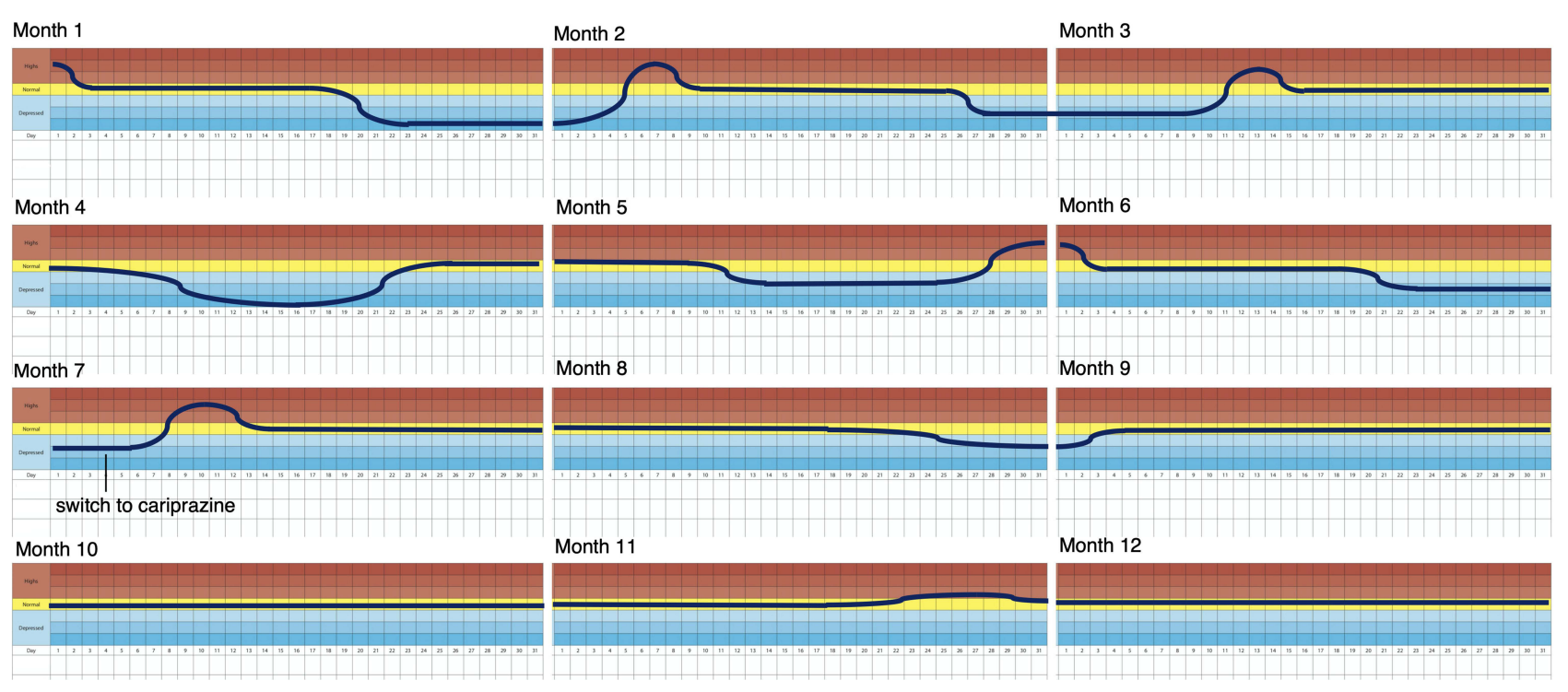

Figure 2 Patient's daily mood chart over the last twelve months.

of three mood stabilizers not showing better outcomes compared to combination of two. ${ }^{18}$ While depressive episodes often represent the major source of suffering for these patients, ${ }^{19}$ several researchers recommend avoiding

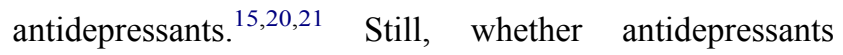
should be allowed in patients with RC is unclear, since they have been shown to destabilize patients with RC in some studies, but not in others. ${ }^{22}$

Cariprazine is approved by the $\mathrm{EMA}^{3}$ for the treatment of schizophrenia in adult patients. Due to its unique pharmacodynamic profile $\left(\mathrm{D}_{3}\right.$-preferring $\mathrm{D}_{3} / \mathrm{D}_{2}$ receptor partial agonism, $5-\mathrm{HT}_{1 \mathrm{~A}}$ receptor partial agonism, and $5-\mathrm{HT}_{2 \mathrm{~B}}$ and $5-\mathrm{HT}_{2 \mathrm{~A}}$ receptor antagonism), cariprazine has been proven to exert a significant effect on both negative and cognitive symptoms. Moreover, a substantial body of data showed its mood stabilizing properties, leading to $\mathrm{FDA}^{2}$ approvalof cariprazine for the treatment of manic, mixed and depressive episodes associated with BD.

Case 3 showed a repetitive pattern of RC mood episodes, which remained unmodified after combination of a stable valproate regimen with several anticonvulsants and second-generation antipsychotics. She also complained about treatment-induced constipation and cognitive impairment. Targeting the lack of tolerability, Case 3 was switched to cariprazine, additionally achieving a prolonged stabilization of mood. The understanding of mechanisms uniquely underlying $\mathrm{RC}$ course of $\mathrm{BD}$ is poor, with most literature considering $\mathrm{RC}$ as a late-stage $\mathrm{BD}$, largely influenced by environmental factors, including those related to psychopharmacological treatment. ${ }^{15}$ One possible interpretation of our case is related to cariprazine's unique receptor binding profile and to the dopaminergic hypothesis of BD. Along with this hypothesis, faulty homoeostasis between dopamine transporter and $D_{2} / D_{3}$ receptors in the striatum may underlie depressive and manic phases of the illness. ${ }^{23}$ In this framework, cariprazine $D_{2} / D_{3}$ partial antagonism, which theoretically acts depending on the current concentration of dopamine, might have a more thorough effect compared to full antagonists, especially in cases showing very high mood instability as in RC BD. On the other hand, cariprazine has been shown to have a significant procognitive effect, which is thought to be associated with its high $\mathrm{D}_{3}$ affinity. As cognitive impairment after resolution of a mood episode is among the most frequent residual symptoms, which is in turn a robust correlate of rapid relapse and more chronic course of $\mathrm{BD},{ }^{24,25}$ we may also hypothesize that the prompt improvement in cognitive symptoms exerted a beneficial effect on the overall course of the mood cycle of Case 3.

\section{Conclusion}

The present paper aimed to provide real-world insight about the use of cariprazine in specific clinical conditions that might deserve further exploration in upcoming research. Three main points summarize our clinical series. First, when cariprazine is administered targeting negative symptoms of schizophrenia, a beneficial effect may take several months to arise. While differential change in negative symptoms was already significant after 3 months of cariprazine 
treatment in Németh et al's controlled trial, ${ }^{11}$ clinicians' experience already suggested extending the follow-up period up to 6 months before further decision about treatment is made. $^{12}$ Second, augmentation with cariprazine may improve symptoms in patients with refractory schizophrenia already treated with clozapine. Even though clinical guidelines usually argue against the long-term use of antipsychotic polypharmacy, clozapine augmentation is somewhat contemplated for patients with more refractory schizophrenia, ${ }^{13}$ and cases with beneficial cariprazine addon have been previously described. ${ }^{14}$ Noteworthy, clozapine and cariprazine profiles may be especially compatible in order to further improve negative and positive symptoms while they prevent the enhancement of cardiometabolic toxicity. Last, beyond $\mathrm{FDA}^{2}$ licensed use of cariprazine for treating mood episodes during the course of BD, no studies have been conducted in heterogeneous populations, such as rapid cycling bipolar patients, who are generally neglected by controlled clinical trials, while accounting for significant treatment resistance and morbidity. ${ }^{24}$ From this perspective, besides adding to extend literature about the clinical use of cariprazine, our case series provides some hints about novel therapeutic applications of cariprazine to be tested in future tailored studies.

\section{Ethics Statements}

We obtained institutional approval from ethics committees of Azienda Socio Sanitaria Territoriale of Lodi and Azienda Socio Sanitaria Territoriale Fatebenefratelli Sacco of Milan to publish these cases.

\section{Informed Consent}

Consent for the publication of the cases was obtained from patients.

\section{Disclosure}

Recordati provided financial support for the writing of this article. Dr Giancarlo Cerveri reports grants from Recordati, during the conduct of the study. The authors report no other conflicts of interest in this work.

\section{References}

1. Misiak B, Bieńkowski P, Samochowiec J. Cariprazine - a novel antipsychotic drug and its place in the treatment of schizophrenia Psychiatr Pol. 2018;52:971-981. doi:10.12740/PP/OnlineFirst/80710

2. Food and Drugs Administration. Available from: https://www.access data.fda.gov/drugsatfda_docs/label/2019/204370s006lbl.pdf. Accessed November 16, 2021.
3. European medicines agency reagila assessment report; 2017. European Medicines Agency. Available from: https://www.ema. europa.eu/en/documents/overview/reagila-epar-summary-public_en. pdf. Accessed November 16, 2021.

4. Ringen PA, Reponen EJ, Vedal TSJ, et al. Predictors for antipsychotic dosage change in the first year of treatment in schizophrenia spectrum and bipolar disorders. Front Psychiatr. 2019;10:649. doi:10.3389/ fpsyt.2019.00649

5. Gilbert P, Harris MJ, McAdams LA. Neuroleptic withdrawal in schizophrenic patients: a review of the literature. Arch Gen Psychiatr. 1995;52(3):173-188. doi:10.1001/archpsyc.1995.0395015 0005001

6. Campbell RH, Diduch M, Gardner KN, et al. Review of cariprazine in management of psychiatric illness. Ment Health Clin. 2017;7 (5):221-229. doi:10.9740/mhc.2017.09.221

7. Fountoulakis KN, Kontis D, Gonda X, et al. A systematic re- view of the evidence on the treatment of rapid cycling bipolar disor- der. Bipolar Disord. 2013;15:115-137. doi:10.1111/bdi.12045

8. Gigante AD, Barenboim IY, Dias RD, et al. Psychiatric and clinical correlates of rapid cycling bipolar disorder: a cross-sectional study. Braz J Psychiatr. 2016;38(4):270-274. doi:10.1590/1516-4446-20151789

9. Cerveri G, Gesi C, Mencacci C. Pharmacological treatment of negative symptoms in schizophrenia: update and proposal of a clinical algorithm. Neuropsychiatr Dis Treat. 2019;15:1525-1535. doi:10.21 47/NDT.S201726

10. Németh G, Laszlovszky I, Czobor P, et al. Cariprazine versus risperidone monotherapy for treatment of predominant negative symptoms in patients with schizophrenia: a randomised, double-blind, controlled trial. Lancet. 2017;389(10074):1103-1113. doi:10.1016/ S0140-6736(17)30060-0

11. Németh B, Molnár A, Akehurst R, et al. Quality-adjusted life year difference in patients with predominant negative symptoms of schizophrenia treated with cariprazine and risperidone. J Comp Eff Res. 2017;6(8):639-648. doi:10.2217/cer-2017-0024

12. Fagiolini A, Alcalá JÁ, Aubel T, et al. Treating schizophrenia with cariprazine: from clinical research to clinical practice. Real world experiences and recommendations from an International Panel. Ann Gen Psychiatr. 2020;19:55. doi:10.1186/s12991-020-00305-3

13. Lehman AF, Lieberman JA, Dixon LB, et al. Practice guideline for the treatment of patients with schizophrenia, second edition. Am J Psychiatr. 2004;161:1-56.

14. De Berardis D, Rapini G, Olivieri L, et al. Cariprazine add-on in inadequate clozapine response: a report on two cases. Clin Psychopharmacol Neurosci. 2021;19(1):174-178. doi:10.9758/cpn. 2021.19.1.174

15. Carvalho AF, Dimellis D, Gonda X, et al. Rapid cycling in bipolar disorder: a systematic review. J Clin Psychiatr. 2014;75(6):578-586. doi:10.4088/JCP.13r08905

16. Furio MA, Popovic D, Vieta E, et al. Characterization of rapid cycling bipolar patients presenting with major depressive episode within the BRIDGE-II-MIX study. Bipolar Disord. 2020. doi:10. 1111/bdi.12994

17. Calabrese JR, Suppes T, Bowden CL, et al. A double-blind, placebo-controlled, prophylaxis study of lamotrigine in rapid-cycling bipolar disorder. Lamictal 614 Study Group. J Clin Psychiatr. 2000;61(11):841-850. doi:10.4088/JCP.v61n1106

18. Kemp DE, Gao K, Fein EB, et al. Lamotrigine as add-on treatment to lithium and divalproex: lessons learned from a double-blind, placebo-controlled trial in rapid-cycling bipolar disorder. Bipolar Disord. 2012;14(7):780-789. doi:10.1111/bdi. 12013

19. Koukopoulos A, Sani G, Koukopoulos AE, et al. Duration and stability of the rapid-cycling course: a long-term personal follow-up of 109 patients. J Affect Disord. 2003;73(1-2):75-85. doi:10.1016/ S0165-0327(02)00321-X 
20. Coryell W, Solomon D, Turvey C, et al. The long-term course of rapid-cycling bipolar disorder. Arch Gen Psychiatr. 2003;60 (9):914-920. doi:10.1001/archpsyc.60.9.914

21. El-Mallakh RS, Vöhringer PA, Ostacher MM, et al. Antidepressants worsen rapid-cycling course in bipolar depression: a STEP-BD randomized clinical trial. J Affect Disord. 2015;184:318-332. doi:10.1016/j.jad.2015.04.054

22. Yatham LN, Kennedy SH, Parikh SV, et al. Canadian Network for Mood and Anxiety Treatments (CANMAT) and International Society for Bipolar Disorders (ISBD) 2018 guidelines for the management of patients with bipolar disorder. Bipolar Disord. 2018;20(2):97-170. doi:10.1111/bdi.12609
23. Ashok A, Marques T, Jauhar S, et al. The dopamine hypothesis of bipolar affective disorder: the state of the art and implications for treatment. Mol Psychiatr. 2017;22:666-679. doi:10.1038/mp.2017.16

24. Judd LL, Schettler PJ, Akiskal HS, et al. Residual symptom recovery from major affective episodes in bipolar disorders and rapid episode relapse/recurrence. Arch Gen Psychiatr. 2008;65(4):386-394. doi:10.1001/archpsyc.65.4.386

25. Vrabie M, Marinescu V, Talaşman A, et al. Cognitive impairment in manic bipolar patients: important, understated, significant aspects. Ann Gen Psychiatr. 2015;14:41. doi:10.1186/s12991-015-0080-0

\section{Publish your work in this journal}

Neuropsychiatric Disease and Treatment is an international, peerreviewed journal of clinical therapeutics and pharmacology focusing on concise rapid reporting of clinical or pre-clinical studies on a range of neuropsychiatric and neurological disorders. This journal is indexed on PubMed Central, the 'PsycINFO' database and CAS, and is the official journal of The International Neuropsychiatric Association (INA). The manuscript management system is completely online and includes a very quick and fair peer-review system, which is all easy to use. Visit http://www.dovepress.com/testimonials.php to read real quotes from published authors. 